\title{
CARACTERIZAÇÃO QUÍMICA E MINERALÓGICA DOS RESÍDUOS DA MINERAÇÃO DE GIPSITA NO SEMIÁRIDO PERNAMBUCANO
}

\author{
K. K. S. MELO ${ }^{1}$, A. P. C LIMA ${ }^{2}$, M. C. SANTANA ${ }^{3}$, V. C. P. ANDRADE ${ }^{4}$, A. L. C. BRAGA ${ }^{5}$, K. V. CORREIA \\ $1,2,3,4,6$ Programa de Pós Graduação em Engenharia Mineral - Universidade Federal de Pernambuco \\ keylakmelo@hotmail.com \\ ${ }^{5}$ Universidade Federal do Sul e Sudeste do Pará \\ luiza.coelho@unifesspa.edu.br
}

Submetido 20/10/2017 - Aceito 29/11/2017

DOI: $10.15628 /$ holos.2017.5626

\begin{abstract}
RESUMO
Uma das principais fontes de impacto ambiental relacionadas às atividades de extração de gipsita são as pilhas de resíduos, sistematicamente dispostas na área de influência direta do empreendimento mineiro, constituídas por finos oriundos do desmonte e pela parcela de material estéril retirada na operação de lavra. Por apresentar uma granulometria que limita seu campo de aplicação, se faz necessário conhecer as propriedades dos resíduos e aproveitá-los como uma alternativa à mitigação dos inconvenientes envolvidos em sua disposição, contribuindo, assim, para o desenvolvimento econômico e social do semiárido pernambucano. Sob essa ótica, o presente trabalho tem por objetivo caracterizar química e mineralogicamente os resíduos do minério de gipsita provenientes de áreas de
\end{abstract}

mineração no município de Araripina, Pernambuco. As amostras de resíduos foram caracterizadas através das técnicas de difratometria de raios $X(D R X)$, fluorescência de raios $X$ (FRX) e microscopia eletrônica de varredura (MEV), acoplada ao espectrômetro de raios $X$ por dispersão em energia (EDS). As análises realizadas mostraram que o material apresenta características potenciais para uso como corretivo de solo com teores significativos de cálcio e enxofre, provenientes dos minerais gipsita e calcita. Estes elementos são essenciais à nutrição e fertilidade do solo, portanto, tem-se como prognóstico o aproveitamento integrado dos resíduos da gipsita em produção de mudas florestais para o provimento da recuperação de áreas degradadas, sistemas agroflorestais e produção de matriz energética.

PALAVRAS-CHAVE: Mineração, Resíduo mineral, Sustentabilidade, Gipsita.

\section{CHEMICAL AND MINERALOGICAL CHARACTERIZATION OF WASTE FROM GYPSUM MINING IN THE SEMI-ARID OF PERNAMBUCO}

\begin{abstract}
One of the main sources of environmental impacts related to extraction activities are gypsum waste piles, conventionally disposed in the area of direct influence of the mining venture, they consist of thin materials from blasting and part of waste removed in the mining operation. By offering a granulometry that limits their application range, it is necessary to know the properties of the waste and take advantage of them as an alternative to mitigate the inconvenience involved in their disposal, thus contributing to the economic and social development of the semi-arid of Pernambuco. Under this perspective, the present study aims to characterize mineralogical and chemically the waste of gypsum ore from mining areas in the city of Araripina,
\end{abstract}

Pernambuco. The waste samples were characterized using $x$-ray diffraction techniques (XRD), x-ray fluorescence spectrometry (XRF) and scanning electron microscope (SEM), coupled to an energy-dispersive $x$-ray spectroscopy (EDS). The analyzed sample results show potential characteristics to use gypsum and calcite material to correct the soil acidity, since it contains significant sulfur and calcium grades, highlighting that these elements are essential to soil nutrition and fertility. Therefore, it implies the entire utilization of these fine mineral particles for the purpose to product seedling in order to supply reclamation of degraded area, agroforestry systems and production of energy matrix.

KEYWORDS: Mining, Mining waste, Sustainability, Gypsum. 


\section{INTRODUÇÃO}

A gipsita é um sulfato de cálcio di-hidratado cuja fórmula química é $\mathrm{CaSO}_{4} \cdot 2 \mathrm{H}_{2} \mathrm{O}$. Ela geralmente ocorre associada à anidrita, um sulfato de cálcio anidro $\mathrm{CaSO}_{4}$ que tem pouca expressão econômica (SOBRINHO et al, 2001). Sua composição estequiométrica média é de 32,5\% de óxido de cálcio $(\mathrm{CaO}), 46,6 \%$ de trióxido de enxofre $\left(\mathrm{SO}_{3}\right)$ e $20,9 \%$ de água $\left(\mathrm{H}_{2} \mathrm{O}\right)$.

É um mineral que ocorre em diversas regiões do mundo e suas reservas são abundantes na maior parte dos países produtores. De acordo com o DNPM (2015), no ano de 2014 a produção mundial de gipsita foi estimada em 246 milhões de toneladas (Mt), um aumento de apenas 0,4\% em relação ao ano de 2013. A China foi apontada como a maior produtora mundial de gipsita (132 Mt), representando 53,7\% de toda a produção de 2014, e o Brasil como o maior produtor da América do Sul e o 13으 do mundo, com uma produção de aproximadamente $3,4 \mathrm{Mt}$, representando $1,4 \%$ do total mundial.

O Estado de Pernambuco possui reservas abundantes de gipsita na região do Sertão do Araripe, envolvendo os Municípios de Araripina, Bodocó, Ipubi, Ouricuri e Trindade e é responsável por $95 \%$ da produção brasileira. As jazidas do Araripe são consideradas as de melhor qualidade de minério do mundo e apresentam excelentes condições de mineração (relação estéril/minério e geomorfologia da jazida) (CETEM, 2005).

O grande interesse pela gipsita é atribuído a uma característica peculiar que consiste na facilidade de desidratação e reidratação. $O$ mineral perde $3 / 4$ da água de constituição durante 0 processo de calcinação, convertendo-se a um sulfato hemidratado de cálcio $\left(\mathrm{CaSO}_{4} \cdot 1 / 2 \mathrm{H}_{2} \mathrm{O}\right)$ que, quando misturado com água, pode ser moldado e trabalhado antes de endurecer e adquirir a consistência mecânica de forma estável hidratada (CETEM, 2005).

Sua utilização pode ser na forma natural, sendo bastante utilizada na indústria do cimento e na forma calcinada, conhecida como gesso. Este tem um campo de aplicação bastante variado que vai desde a indústria da construção civil até a de material ortopédico, dental, corretivo de pH do solo na agricultura, etc.

O Polo Gesseiro do Araripe gera grande lucro com a extração e beneficiamento da gipsita, promovendo o desenvolvimento econômico regional com geração de empregos diretos e indiretos; contudo, a gipsita produz grandes quantidades de resíduos, tanto na sua extração quanto na fabricação dos seus produtos (LIMA et al, 2016).

Dentre umas das principais fontes de impacto ambiental alusivo a esta atividade está a grande geração e concentração dos resíduos de gipsita dispostos em pilhas e descartados no interior da cava em operação e em áreas adjacentes. Trata-se de um fino com alto teor de pureza oriundo da operação de desmonte, inerte, sem ter passado por qualquer tipo de beneficiamento. Contudo, sua granulometria fina limita seu campo de aplicação.

Esses resíduos inorgânicos ficam expostos à ação do vento e promovem a poluição do ar por particulados em suspensão. De acordo com Fernandes e Barbosa (2011), a geração de poeira provocada pela extração da gipsita é considerada um dos fatores agravantes ao ambiente no município de Araripina, ocasionando alterações significativas às condições de vida local. Esta poeira exerce um amplo espectro de problemas à saúde dos trabalhadores que estão sob áreas de influência direta do empreendimento, além da população circunvizinha, provocando desde efeitos irritativos nos olhos até doenças pulmonares crônicas. 
Segundo Menezes et al. (2007), as premissas do desenvolvimento sustentável objetivam a minimização do descarte dos materiais oriundos das atividades antropogênicas, o que corresponde às diretrizes da Política Nacional de Resíduos Sólidos, a qual contempla dentre suas diretrizes o aproveitamento racional e reciclagem dos resíduos da mineração como instrumentos de mitigação dos impactos associados a esta atividade (BRASIL, 2010).

Portanto, o aproveitamento integrado dos resíduos junto à cadeia produtiva agroflorestal pode ser uma medida de mitigação dos impactos ambientais atribuídos a quantidade de resíduo descartado no ambiente. Entre às vantagens deste investimento está a agregação de valor econômico a um material até então sem aproveitamento, movimentação da economia local, esgotamento de reserva de matéria prima, redução de custos operacionais e destinação final.

Neste contexto, a presente pesquisa teve por objetivo caracterizar química e mineralogicamente os resíduos de gipsita oriundos de áreas de mineração no município de Araripina, Pernambuco.

\section{METODOLOGIA}

Neste estudo foi analisado o resíduo de gipsita proveniente de uma jazida localizada no município de Araripina, extremo oeste do Estado de Pernambuco, mesorregião Sertão, coordenadas geográficas S $07^{\circ} 44^{\prime} 28.1^{\prime \prime}$ e W $040^{\circ} 27^{\prime} 51.0^{\prime \prime}$ e elevação de 516 metros. Trata-se de um material de fina granulometria oriundo da operação do desmonte, descartado e acumulado em pilhas. As amostras foram coletadas aleatoriamente em pilhas de resíduos dispostas na cava de mineração. Nos pontos de coleta foram obtidas amostras simples para compor uma amostra composta. As amostras foram homogeneizadas em lona e posteriormente quarteadas, segundo método de pilha cônica, garantindo a retirada de uma amostra representativa em quantidade suficiente para realização dos ensaios requeridos.

A amostra final foi analisada mediante o método do pó, empregando-se um difratômetro de raios $X$ Shimadzu XRD-7000 com radiação CuKa, tensão de $40 \mathrm{KV}$, corrente de $30 \mathrm{~mA}$, tamanho de passo de $0,02{ }^{\circ} 2 \theta$ e tempo por passo de $1 \mathrm{~s}$, com velocidade de varredura de $20\left({ }^{\circ} 2 \theta\right) / \mathrm{min}$, com ângulo de $2 \theta$ percorrido de $10^{\circ}$ a $60^{\circ}$.

Para análise química, uma porção de amostra foi secada em estufa a $110^{\circ} \mathrm{C}$. Uma porção de amostra seca foi levada a uma mufla a $1000^{\circ} \mathrm{C}$ por 2 horas para determinação da perda ao fogo. Uma outra porção de amostra seca foi prensada em cápsulas de alumínio a 30 toneladas de força. A pastilha prensada foi analisada em espectrômetro de fluorescência de raios X Rigaku modelo ZSX Primus II, equipado com tubo de Rh e 7 cristais analisadores. Os resultados da varredura semiquantitativa foram recalculados para $100 \%$ após a incorporação do valor da perda ao fogo. Os resultados estão expressos em peso\%.

A morfologia dos grãos foi verificada com o uso de microscopia eletrônica de varredura, acoplado ao EDS (HITACHI, Swift ED3000) que permitiu determinar a composição semiquantitativa dos minerais. A amostra na forma de pó foi fixada ao porta-amostra por uma fita adesiva de carbono. As micrografias foram obtidas no microscópio eletrônico de varredura da marca HITACHI, modelo TM3000, em modo de alto vácuo por elétrons retroespalhados. 


\section{RESULTADOS E DISCUSSÕES}

De uma forma geral, através da técnica de difração de raios $X$ pôde-se identificar e classificar os minerais presentes na amostra de resíduo de gipsita, confirmando-se que a mesma é

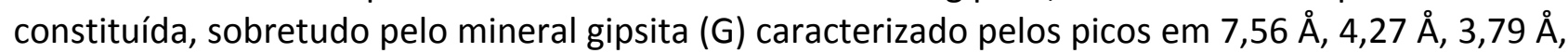
$3,059 \AA, 2,216 \AA, 1,898 \AA$ e $1,778 \AA$ associados respectivamente aos planos (020), (12 $\overline{1}),(031)$,

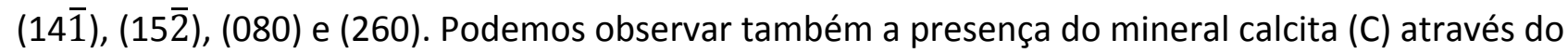
pico $2,88 \AA$ correspondente ao plano (006) e pelo argilomineral caulinita (K) com pico em $2,63 \AA$ referente ao plano (130), conforme representados no difratograma da Figura 1.

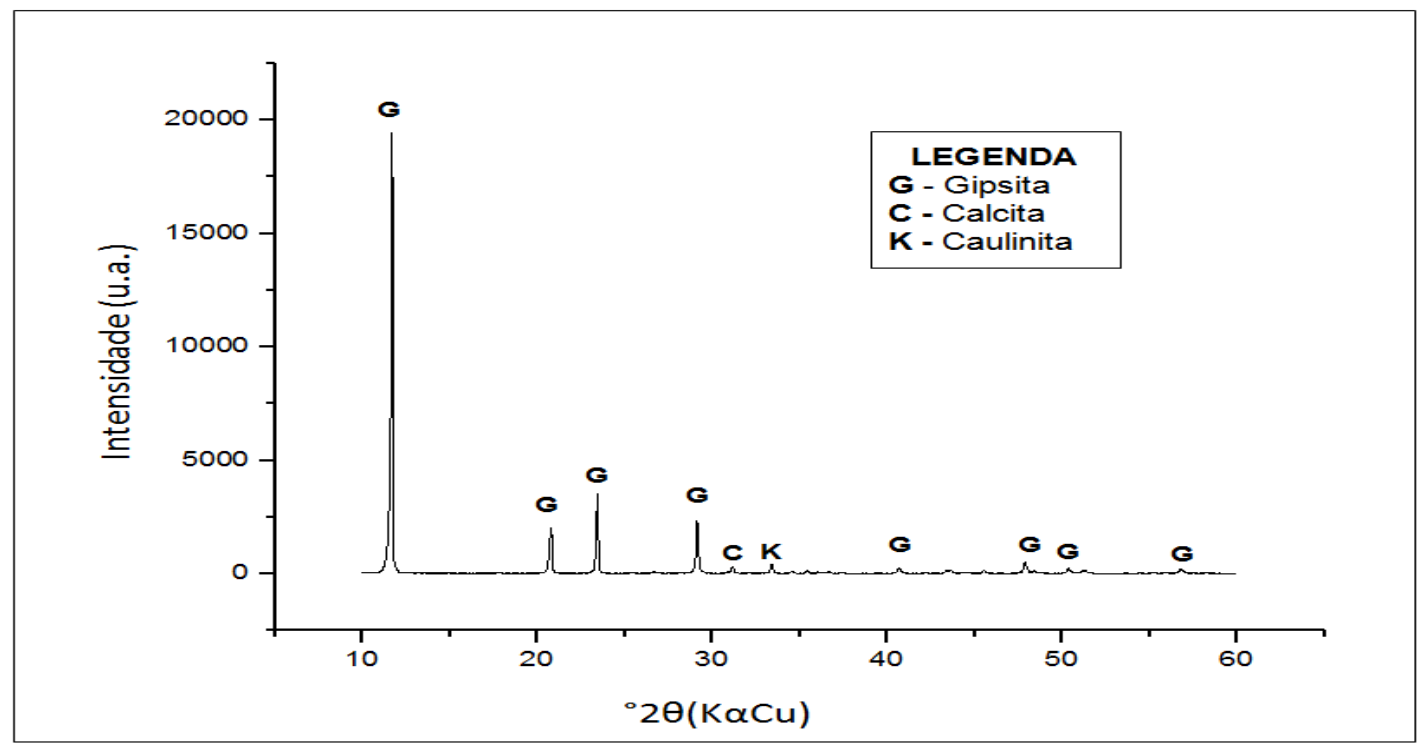

Figura 1:Resultados da análise de DRX da amostra de resíduo de gipsita.

A amostra foi também avaliada a partir da análise química semi-quantitativa por espectrometria de fluorescência de raios $X$. A composição química do resíduo de gipsita, discriminada na Tabela 1, indica a presença de pequenos traços decomponentes como $\mathrm{TiO}_{2}, \mathrm{MnO}_{\text {, }}$ SrO entre outros. No entanto, diante das frações mássicas atribuídas as impurezas presentes, pôde-se observar a elevada quantidade de cálcio na amostra.

Tabela 1: Resultado da análise química semi-quantitativa da amostra de resíduo de gipsita.

\begin{tabular}{c|c|c|c}
\hline Componente & $\begin{array}{c}\text { Porcentagem } \\
\text { (\%) }\end{array}$ & Componente & $\begin{array}{c}\text { Porcentagem } \\
\text { (\%) }\end{array}$ \\
\hline $\mathrm{Na}_{2} \mathrm{O}$ & 0,03 & $\mathrm{TiO}_{2}$ & 0,06 \\
$\mathrm{MgO}$ & 0,42 & $\mathrm{MnO}$ & 0,02 \\
$\mathrm{Al}_{2} \mathrm{O}_{3}$ & 1,34 & $\mathrm{Fe}_{2} \mathrm{O}_{3}$ & 0,48 \\
$\mathrm{SiO}_{2}$ & 3,44 & $\mathrm{CuO}$ & 0,00 \\
$\mathrm{P}_{2} \mathrm{O}_{5}$ & 0,03 & $\mathrm{ZnO}$ & 0,00 \\
$\mathrm{SO}_{3}$ & 39,17 & $\mathrm{Rb}_{2} \mathrm{O}$ & 0,00 \\
$\mathrm{~K}_{2} \mathrm{O}$ & $\mathrm{SrO}$ & 0,12 \\
$\mathrm{COO}$ & 0,23 & $\mathrm{P} . \mathrm{F}$. & 27,22 \\
\hline \multicolumn{2}{|c}{ TOTAL $=100,00$} \\
\hline
\end{tabular}


O teor de óxido de silício $\left(\mathrm{SiO}_{2}\right)$ verificado na amostra de acordo com Souza Santos (1989), pode estar associado à presença de silicatos, que são os argilominerais, micas e os feldspatos encontrados em baixas concentrações. Já o teor de óxido de alumínio $\left(\mathrm{Al}_{2} \mathrm{O}_{3}\right)$ apresentado, devese a sua combinação formando argilominerais, como a caulinita evidenciada na análise de difração. O teor de óxido de cálcio $(\mathrm{CaO})$ existente na amostra, deve-se à existência da calcita e gipsita minerais também identificados através do difratograma. $\mathrm{O}$ teor de óxido de enxofre $\left(\mathrm{SO}_{3}\right)$ é proveniente da gipsita.

A perda ao fogo (P.F.) verificada na amostra dá-se, principalmente, devido às águas dos tipos intercalada, de coordenação e zeolítica, à água de hidroxilas de argilominerais e também de hidróxidos tais como $\mathrm{Ca}(\mathrm{OH})_{2}$ e o $\mathrm{Al}(\mathrm{OH})_{3}$. No entanto segundo Souza Santos (1989), os componentes voláteis como sulfetos, sulfatos, carbonatos e matéria orgânica, quando presentes também são incluídos nessa determinação.

$\mathrm{Na}$ Figura 2a é apresentada a microestrutura de partículas do resíduo de gipsita. Na microimagem é possível observar as diferentes formas dos cristais: oval, angular, hexagonal plano, retangular, entre outros, com superfície lisa e irregular.

A caracterização morfológica é importante, sendo um passo fundamental para se conhecer determinado minério, pois caracteriza as microestruturas e identifica seus mecanismos formadores.

A análise semi-quantitativa do resíduo de gipsita, obtida através de análise EDS, conforme ilustra a Figura $2 \mathrm{~b}$, apresenta os principais elementos químicos que constituem a amostra. A composição química geral da superfície da amostra estudada demonstra a presença dos elementos cálcio, em maior quantidade, alumínio, silício, oxigênio referentes à composição química da caulinita e calcita, minerais evidenciados na análise por difração, e o enxofre proveniente do sulfato de cálcio, confirmando potencial fonte de cálcio e enxofre que o material apresenta.

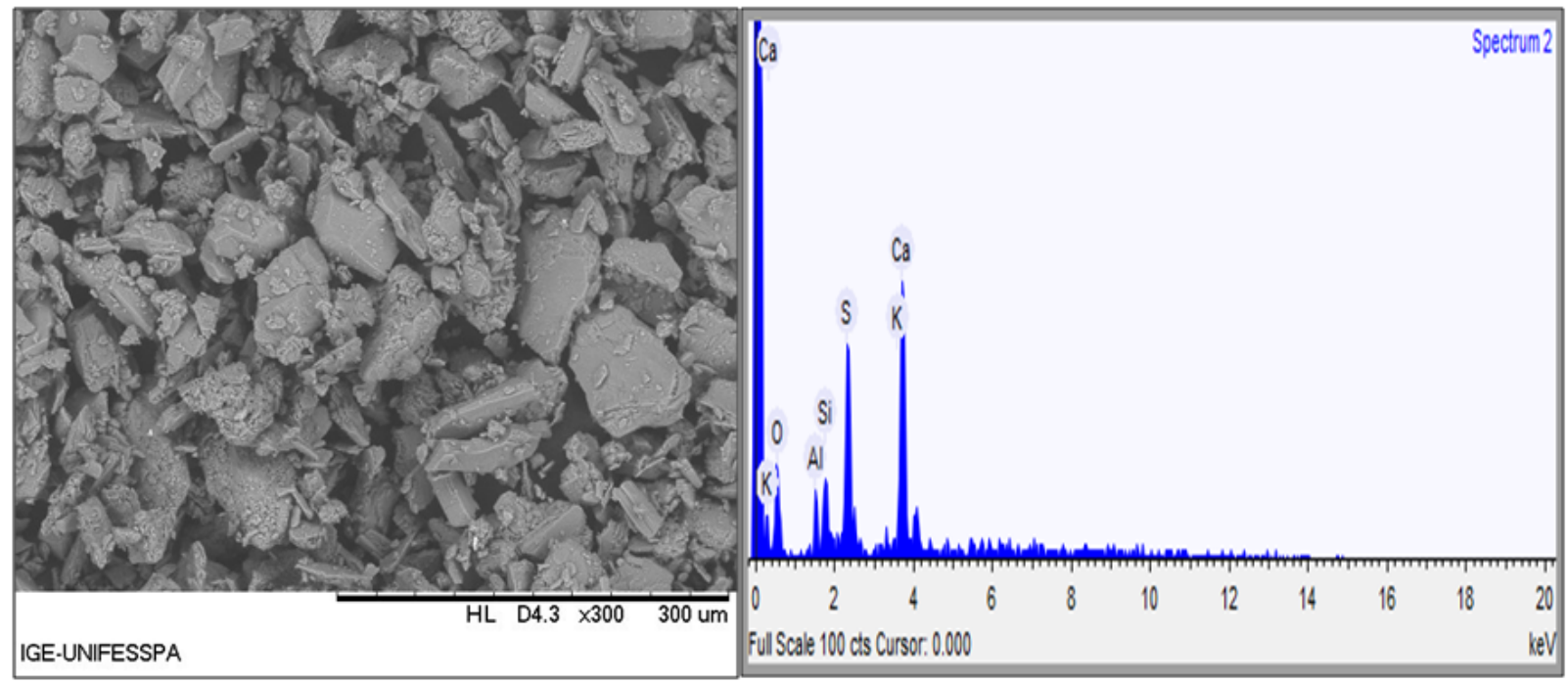

a)

b)

Figura 2: a) Microestrutura de partículas do resíduo de gipsita -b) Composição química dos principais elementos que compõem a amostra, evidenciados no espectro de EDS. 
A presença, também, desses elementos no resultado da fluorescência de raios $X$, onde os valores apresentados foram 39,17 $\left(\mathrm{SO}_{3}\right), 27,44(\mathrm{CaO}), 3,44\left(\mathrm{SiO}_{2}\right), 1,34\left(\mathrm{Al}_{2} \mathrm{O}_{3}\right)$ servem para corroborar ainda mais os resultados do DRX e do MEV apresentados.

A gipsita in natura aplicada ao solo pode promover o aumento de teores de cálcio e consequentemente a redução dos teores de alumínio, ocasionando um maior aprofundamento das raízes pela redução de compactação do solo e dos seus efeitos tóxicos, auxiliando na absorção de água e nutrientes contidos no solo. A atuação desses resíduos pode ativar a vida microbiana do solo e aumentar a resistência das plantas aos períodos de estiagem e fitopatologias.

Segundo Pavan et al. (1982), a toxidez do alumínio é um dos principais fatores que prejudicam o crescimento das plantas afetando severamente a parte aérea e radicular. Assim, acredita-se que a utilização deste material, bem como sua solubilização e as reações que ocorrem no solo possam promover a melhoria das condições superficiais e subsuperficiais, aumentando os teores de cálcio em profundidade e favorecendo o aprofundamento do sistema radicular.

Por outro lado, o uso desses resíduos pode ser intensificado em solos sódicos como fonte de cálcio na solução para substituir o sódio no complexo de troca e permitir a lixiviação do sulfato de sódio na água de drenagem, resultando em maior floculação da argila e aumento da permeabilidade do solo. Ruiz et al. (2006) constataram, trabalhando com solos salino-sódicos da Paraíba, que o corretivo utilizado (gesso) como fonte de cálcio proporcionou maiores valores para a taxa de infiltração em relação às amostras controle. $O$ sódio é um elemento que promove a dispersão do solo, com deterioração da estrutura, entupimento dos poros maiores e diminuição da condutividade hidráulica (Romero-Aranda et al., 1998).

Através das análises químicas por FRX e EDS, pôde-se destacar também a presença significativa de enxofre na amostra estudada, na qual é encontrada na forma inorgânica. Dentre os sulfatos encontrados na natureza, a gipsita é um dos mais importantes, sendo encontrada em muitas formações geológicas em camadas de extensões consideráveis. Neste contexto, a especificidade de atuação de grupos de microrganismos, temperatura, aeração, umidade e pH do solo irão promover a mineralização do enxofre em ânion sulfato $\left(\mathrm{SO}_{4}{ }^{-2}\right)$, forma disponível para as plantas(SUMNER, 1995).

\section{CONCLUSÃO}

O resíduo de gipsita in natura do município de Araripina apresentou de acordo com os resultados obtidos nas análises de caracterização química e mineralógica, teores significativos de cálcio e enxofre, provenientes dos minerais gipsita e calcita, sendo estes elementos essenciais à nutrição e fertilidade do solo.

Por fim, em atendimento aos resultados descritos, tem-se como prognóstico o incentivo ao aproveitamento deste insumo mineral em ensaios como corretivo de solo na implementação de cultivos diversos, no tocante a gestão e reabilitação das áreas de bota fora da mineração com utilização de espécies florestais nativas, para a produção de material energético, implantação de sistemas agroflorestais, recomposição da paisagem, promovendo assim a sustentabilidade, recuperação ambiental e crescimento socioeconômico das regiões oriundas e circunvizinhas de atividades de mineração. 


\section{REFERÊNCIAS}

BRASIL.(2010). Lei N 12.305 de 02 de agosto de 2010 - Política Nacional de Resíduos Sólidos (PNRS).

CETEM. (2005).Rochas e Minerais Industriais - Usos e Especificações. Luz, A. B. e Lins, F. F. (Editores). 449- 470

DNPM. (2015). Sumário Mineral. Brasília, V. 35. p. 72.

FERNANDES, M. F., BARBOSA, M. P. (2011). Aplicações dos indicadores socioeconômicos e ambientais no modelo DPSIR (Força Motriz/Pressão/Estado/Impacto/Resposta) e influências na desertificação nos municípios de Araripina-PE, Crato e Barbalha-CE e Marcolândia-PI. Revista Brasileira de Geografia Física 04 (2011) 722-737 - UFPE. 730 p.

ROMERO-ARANDA, R. MOYA, J.L. TADEO, F.R. LAGAZ, F. PRIMO-MILLO E \& TALON, M. (1998). Physiological and anatomical disturbances induced by chloride salts in sensitive and tolerant citrus: Beneficial and detrimental effects of cations. Plant, Celland Environment, 21:12431253.

RUIZ, H. A.; SAMPAIO, R. A.; OLIVEIRA, M. de; FERREIRA, P. A. (2006).Características físicas de solos salino-sódicos submetidos a parcelamento da lâmina de lixiviação. Journal of Soil Science and Plant Nutrition, v.6, p.1-12.

SOBRINHO, A. C. P. L.; AMARAL, A. J. R.; DANTAS, J. O. C.; DANTAS, J. R. A. (2001). Balanço Mineral Brasileiro - Gipsita. Disponível em: <http://www.dnpm.gov.br/dnpm/paginas/balancomineral/arquivos/balanco-mineral-brasileiro-2001> Acesso em 20 mai 2017.

SOUZA SANTOS, P. PAVAN, M.A.; BINGHAM, F.T.; PRATT, P.F. (1982). Toxicity of aluminium to coffee in ultisols and oxisols amended with $\mathrm{CaCO}_{3}, \mathrm{MgCO}_{3}$, and $\mathrm{CaSO}_{4} .2 \mathrm{H}_{2} \mathrm{O}$. Soil Science Society of America Journal, Madison, v.46. p.1201-1207.

SUMNER, M.E. (1995). Amelioration of subsoil acidity with minimum disturbance. In: JAYAWARDANE, N.S.; STEWART, B.A., eds. Subsoil management techniques. Athens, GA, Lewis Publishers, p.147-185 